\title{
A NUMERICAL SCHEME FOR SOLVING CREEPING FLOWS
}

\author{
H. A. Navarro, \\ Instituto de Geociências e Ciências Exatas, \\ IGCE-UNESP, Av. 24-A, 1515 Bela Vista, \\ Cx.P. 178, 13506-900, Rio Claro, SP, Brasil \\ helio@rc.unesp.br \\ V. G. Ferreira, \\ Instituto de Ciências Matemáticas e de \\ Computação, ICMC-USP, Av.do Trabalhador \\ São-Carlense, 400, Cx.P. 668, 13560-970, São \\ Carlos, SP, Brasil \\ pvgf@1cad.icmc.usp.br
}

\author{
ABSTRACT \\ This work shows an extension of the generalized Peaceman and \\ Rachford alternating-direction implicit (ADI) scheme for simulating \\ two-dimensional fluid flows at low Reynolds numbers. The \\ conservation equations are solved in stream function - vorticity \\ formulation. We compare the ADI and generalized ADI schemes, and \\ show that the latter is more efficient to simulate a creeping flow. \\ Numerical results demonstrating the applicability of this technique are \\ also presented.
}

Keywords: creeping flow, alternating direction implicit scheme, stream function - vorticity.

\section{INTRODUCTION}

For low and high Reynolds numbers problems, it is difficult to obtain numerical solutions due to the inertia and viscous terms of the conservation laws. The stream function-vorticity formulation is a method for solving incompressible viscous flow problems. Peaceman and Rachford (1955) proposed an alternating-direction implicit scheme (PR-ADI) for solving parabolic differential equations. For low Reynolds number problems, this method converges slowly to the exact solution (Dean and Glowinski, 1993). To overcome this drawback of the PR-ADI scheme, a new method called $\theta$ scheme has been developed (Dean and Glowinski, 1993; Glowinski, 1987). In this method, there are split parameters $\alpha$ and $\beta$ such that $\beta$ must be less than $\alpha$. In the usage of the ADI scheme in two-dimensional problems, it often occurs that $\alpha=\beta=1 / 2$. Dai (1997) proposed a new ADI scheme for solving two-dimensional parabolic equation based on the idea of regularized difference schemes (Samarskii and Vabishchevich, 1994). Dai's two-level difference scheme generalizes the PR-ADI scheme, and it is called generalized. It also overcomes the drawback of the PR-ADI scheme.

The present study applies the generalized Dai scheme to solve incompressible viscous flow problems for low Reynolds numbers. These flows refer to fluid motions that are dominated by viscosity and are often at the intersection of research problems in biology, chemistry, engineering, and physics. The influence of viscosity becomes more important when motions concern either progressively smaller objects or slower flows. The primary reason for this is that as the surface area per unit volume of the object increases, the frictional contact with the fluid becomes increasingly more important. In particular, the fluid flow in a rectangular region is considered in this work. Several authors have investigated the cavity problem, where the motion is driven by the uniform translation of the top wall. Burggraf (1966) investigated the analytical and numerical solutions of the flow in this domain. Pan and Acrivos (1967) studied the steady flow in rectangular cavities showing experimental results. Guia et al. (1982) presented solutions for stream function - vorticity formulation of two-dimensional incompressible NavierStokes equations using a multigrid technique. The present study complements these investigations in two aspects: (a) analyzing low Reynolds number problems; and (b) showing a generalized scheme for this kind of flow. A time-stepping algorithm based on an extension of the generalized Peaceman and Rachford alternatingdirection implicit scheme for simulating low Reynolds number fluid problems is also presented.

\section{GOVERNING EQUATIONS AND BOUNDARY CONDITIONS}

For a Newtonian incompressible fluid, with constant kinematic viscosity $v$, the Helmholtz vorticity equation takes the form

$$
\frac{\mathrm{D} \omega}{\mathrm{D} t}=(\omega . \nabla) \mathbf{V}+v \nabla^{2} \omega
$$

where $\mathrm{D} / \mathrm{D} t$ is the substantial derivative, $\mathbf{V}$ and $\omega=\nabla \times \mathbf{V}$ are the velocity and vorticity vectors, respectively. In two-dimensional problems, the vorticity is a scalar and the vector potential $\Psi(\mathbf{V}=\nabla \times \Psi)$ is replaced by the stream function $\psi$.

A pseudo-transient approach (Hoffmann and Chiang, 1995; Roache, 1972; Widllund, 1967) for incompressible viscous fluid flow problems is expressed by the following equations

$$
\begin{aligned}
& \frac{\partial \omega}{\partial t}+u \frac{\partial \omega}{\partial x}+v \frac{\partial \omega}{\partial y}=\frac{1}{\operatorname{Re}} \nabla^{2} \omega, \\
& \frac{\partial \psi}{\partial t}-\gamma\left(\nabla^{2} \psi+\omega\right)=0, \\
& u=\frac{\partial \psi}{\partial y},
\end{aligned}
$$


CIÊNCIA/SCIENCE

$$
v=-\frac{\partial \psi}{\partial x},
$$

where $t$ is the time, $u$ and $v$ are the components of velocity along the $x$ and $y$ directions, respectively, $\gamma$ is an arbitrary constant and $\mathrm{Re}=U L / \mathrm{V}$ is the Reynolds number. The Eqs. (2a)-(2d) have been non-dimensionalized by a characteristic velocity $U$, length scale $L$ and kinematic viscosity $v$. If a steady solution exists, it can be obtained numerically if a time marching procedure is carried out until the time-independent boundary conditions force a steady-state. After the computation of the velocity field, if the pressure $p$ is required, one needs to solve an extra Poisson equation.

A driven cavity problem is represented by a rectangular domain $\Omega=\left(0, x_{\max }\right) \times\left(0, y_{\max }\right)$ and the boundary set $\partial \Omega=\left\{\left(x=0,0 \leq y \leq y_{\max }\right),\left(y=0,0 \leq x \leq x_{\max }\right)\right.$, $\left.\left(x=x_{\max }, 0 \leq y \leq y_{\max }\right), \quad\left(y=y_{\max }, 0 \leq x \leq x_{\max }\right)\right\}$. The Cartesian coordinate system is positioned at the origin $\mathrm{O}(x=0, y=0)$. The vector velocity $\mathbf{V}$ is imposed on the top boundary $\left(y=y_{\max }, 0 \leq x \leq x_{\max }\right)$. The aspect ratio $\mathrm{AR}$ is a value given by the relation $y_{\max } / x_{\max }$. Tannehill et al. (1997) present a second order accurate approximation for the vorticity on the boundary. These authors mention that this approximation can lead to unstable calculations at moderate to high Reynolds numbers. In order to have the proposed method working for both high and low Reynolds numbers, we shall be using a first order approximation for the vorticity on the boundary, as described below. A comprehensive review of boundary conditions for the vorticity in the numerical solution of the stream function - vorticity equations is discussed by Napolitano et al. (1999).

On the boundary $\partial \Omega$ of a domain $\Omega$, we prescribe the velocity vector $\mathbf{V}_{\mathbf{b}}$, which can be related to the outer unit normal vector (n) and the unit tangent vector (t) by the expressions

$$
\begin{aligned}
& \mathbf{V}_{b} \cdot \mathbf{t}=f(x), \\
& \mathbf{V}_{b} \cdot \mathbf{n}=g^{\prime}(x) .
\end{aligned}
$$

Using the definition of the stream function, the boundary conditions on the part $\partial \Omega_{x}=\partial \Omega(x, 0)$ of the boundary $\partial \Omega$ are represented by

$$
\begin{aligned}
& \frac{\partial \psi}{\partial y}(x, 0)=+u(x, 0)=\mathbf{V}_{\mathbf{b}} \cdot \mathbf{t}=f(x) . \\
& \frac{\partial \psi}{\partial x}(x, 0)=-v(x, 0)=\mathbf{V}_{\mathbf{b}} \cdot \mathbf{n}=g^{\prime}(x) .
\end{aligned}
$$

A grid point in the domain $\Omega$ is described by $\left(x_{i}\right.$, $\left.y_{j}\right)$, where $x_{i}=i h_{x}, y_{j}=j h_{y}, i=0,1, \ldots, N, j=0,1, \ldots, M, h_{x}$ and $h_{y}$ are the grid sizes such that $N h_{x}=x_{\max }$ and $M h_{y}=y_{\max }$. Integrating the Eq. (4b), with the integrating constant chosen equal to zero, we obtain the stream function on the boundary $\partial \Omega_{\mathrm{x}}$

$$
\psi\left(x_{i}, 0\right)=g\left(x_{i}\right) .
$$

H. A. Navarro and V. G. Ferreira A Numerical Scheme...

For the calculation of the vorticity on the boundary, we need to compute $\frac{\partial^{2} \psi}{\partial x^{2}}(x, 0)$ and $\frac{\partial^{2} \psi}{\partial y^{2}}(x, 0)$ at the grid points $\left(x_{i}, 0\right)$ of the boundary $\partial \Omega_{\mathrm{x}}$. From $(4 \mathrm{~b})$ we obtain

$$
\frac{\partial^{2} \psi}{\partial x^{2}}(x, 0)=g^{\prime \prime}(x)
$$

We write the Taylor expansion to calculate the second derivative of $\psi$ with respect to $y$ as

$$
\begin{aligned}
& \psi\left(x_{i}, y_{1}\right)=\psi\left(x_{i}, 0\right)+\left.h_{y} \frac{\partial \psi}{\partial y}\right|_{\left(x_{i}, 0\right)}+ \\
& \left.\frac{\left(h_{y}\right)^{2}}{2} \frac{\partial^{2} \psi}{\partial y^{2}}\right|_{\left(x_{i}, 0\right)}+O\left(h_{y}^{3}\right)
\end{aligned}
$$

where $y_{1}$ is an specific value in the domain $\Omega$ so that $y_{1}=h_{y}$. Using this equation, one obtains the desired value of the vorticity on the boundary $\partial \Omega_{\mathrm{x}}$, namely

$$
\begin{aligned}
& -\omega\left(x_{i}, 0\right)=\left.\nabla^{2} \psi\right|_{\left(x_{i}, 0\right)}, \\
& \left.\frac{\partial^{2} \psi}{\partial y^{2}}\right|_{\left(x_{i}, 0\right)}=\frac{2}{h_{y}^{2}}\left(\psi_{\left(x_{i}, y_{1}\right)}-g\left(i . h_{x}\right)\right) \\
& -\frac{2}{h_{y}} f\left(i . h_{x}\right)+O\left(h_{y}\right) \\
& \left.\frac{\partial^{2} \psi}{\partial x^{2}}\right|_{\left(x_{i}, 0\right)}=g^{\prime \prime}\left(i . h_{x}\right) .
\end{aligned}
$$

The same analysis could be done on the part

$$
\begin{aligned}
& \partial \Omega_{y}=\partial \Omega(0, y) \quad \text { of the boundary } \partial \Omega, \\
& \partial \Omega_{x}=\partial \Omega\left(x, y_{\max }\right) \text {, and } \partial \Omega_{y}=\partial \Omega\left(x_{\max }, y\right) . \\
& \text { GENERALIZED PEACEMAN-RACHFORD ADI } \\
& \text { SCHEME FOR PARABOLIC EQUATIONS }
\end{aligned}
$$

\section{GENERALIZED PEACEMAN-RACHFORD ADI SCHEME FOR PARABOLIC EQUATIONS}

Dai applied the generalized Peaceman-Rachford ADI scheme only to parabolic differential equations, namely to the problem

$$
\begin{aligned}
& \frac{\partial w}{\partial t}=k \nabla^{2} w, \quad 0 \leq x, y \leq 1, t>0, \\
& w(x, y, 0)=w_{0}(x, y), \quad 0 \leq x, y \leq 1, \\
& w(x, y, t)=u_{\partial \Omega}(x, y), \quad t>0,
\end{aligned}
$$

where $k$ is a positive constant. In this particular problem, the Dai scheme is represented by 
CIÊNCIA/SCIENCE

$$
\begin{aligned}
& \left(1-\varepsilon \frac{k \Delta t}{2 h^{2}} \delta_{x}^{2}\right) \frac{w_{i j}^{n+1 / 2}-w_{i j}^{n}}{\Delta t / 2}=\frac{k}{h^{2}} \delta_{x}^{2} w_{i j}^{n+1 / 2}+, \\
& \frac{k}{h^{2}} \delta_{y}^{2} w_{i j}^{n} \\
& \left(1-\varepsilon \frac{k \Delta t}{2 h^{2}} \delta_{y}^{2}\right) \frac{w_{i j}^{n+1}-w_{i j}^{n+1 / 2}}{\Delta t / 2}=\frac{k}{h^{2}} \delta_{x}^{2} w_{i j}^{n+1 / 2}+, \\
& \frac{k}{h^{2}} \delta_{y}^{2} w_{i j}^{n+1}
\end{aligned}
$$

where h is the grid size, $\Delta t=t^{\mathrm{n}+1}-t^{\mathrm{n}}$ is the time step, $\varepsilon \geq 0$ is a small parameter, and $\delta_{x}^{2}$ and $\delta_{y}^{2}$ are the usual central difference operators. When $\varepsilon=0$, it becomes the PR-ADI scheme. Dai called this scheme the generalized Peaceman-Rachford ADI scheme. It is very well suited for simulating fast transient phenomena and it captures efficiently steady-state solutions of parabolic differential equations (Dai, 1997). The present work applies the same scheme for low Reynolds number problems, which have different characteristics from a single parabolic equation.

\section{STABILITY OF THE PEACEMAN-RACHFORD ADI SCHEME FOR THE VORTICITY EQUATION}

The approximation for the exact solution of the vorticity (Eq.2a), or the stream function (Eq.2b), is $\phi\left(x_{i}\right.$, $\left.y_{j}, n \Delta t\right) \equiv \phi_{i j}{ }^{n}$. Considering Eq. (2a), the PR-ADI scheme can be written in the forms of Eqs. (11a) and (11b):

$$
\begin{aligned}
& \frac{\omega_{i j}^{n+1 / 2}-\omega_{i j}^{n}}{\Delta t / 2}=-\frac{u_{i j}^{n}}{2 h_{x}} \delta_{x} \omega_{i j}^{n+1 / 2}-\frac{v_{i j}^{n}}{2 h_{y}} \delta_{y} \omega_{i j}^{n}+ \\
& \frac{1}{\operatorname{Re} h_{x}^{2}} \delta_{x}^{2} \omega_{i j}^{n+1 / 2}+\frac{1}{\operatorname{Re} h_{y}^{2}} \delta_{y}^{2} \omega_{i j}^{n} \\
& \frac{\omega_{i j}^{n+1}-\omega_{i j}^{n+1 / 2}}{\Delta t / 2}=-\frac{u_{i j}^{n}}{2 h_{x}} \delta_{x} \omega_{i j}^{n+1 / 2}-\frac{v_{i j}^{n}}{2 h_{y}} \delta_{y} \omega_{i j}^{n+1}+ \\
& \frac{1}{\operatorname{Re} h_{x}^{2}} \delta_{x}^{2} \omega_{i j}^{n+1 / 2}+\frac{1}{\operatorname{Re} h_{y}^{2}} \delta_{y}^{2} \omega_{i j}^{n+1}
\end{aligned}
$$

where $\delta_{x}$ and $\delta_{\mathrm{y}}$ are the central difference operators. The components of the velocity $u$ and $v$ are fixed in each time step. Equation (11a) expresses the vorticity at the point $\left(x_{i}, y_{j}, t^{n+1 / 2}\right)$ and Eq. (11b) is related to the vorticity at the point $\left(x_{i}, y_{j}, t^{n+1}\right)$. For a discrete Fourier mode $\omega_{i j}^{n}=\rho^{n}\left(k_{1}, k_{2}\right) e^{I\left(k_{1} x_{i}+k_{2} y_{j}\right)}$, the amplification factor $\rho$ takes the form

$$
\begin{aligned}
& \rho=\frac{1-\lambda_{x}\left(1-\cos k_{1} h_{x}\right)-\mathrm{I} \frac{\sigma_{x}}{2} \sin _{1} h_{x}}{1+\lambda_{x}\left(1-\cos k_{1} h_{x}\right)+\mathrm{I} \frac{\sigma_{x}}{2} \sin _{1} h_{x}} . \\
& \frac{1-\lambda_{y}\left(1-\cos k_{2} h_{y}\right)-\mathrm{I} \frac{\sigma_{y}}{2} \operatorname{sink}_{2} h_{y}}{1+\lambda_{y}\left(1-\cos k_{2} h_{y}\right)+\mathrm{I} \frac{\sigma_{y}}{2} \sin _{2} h_{y}} \\
& \sigma_{x}=\frac{u_{i j}^{n} \Delta t}{h_{x}}, \sigma_{y}=\frac{v_{i j}^{n} \Delta t}{h_{y}},
\end{aligned}
$$

$$
\lambda_{x}=\frac{\Delta t}{\operatorname{Re} h_{x}^{2}}, \lambda_{y}=\frac{\Delta t}{\operatorname{Re} h_{y}^{2}},
$$

where $\mathrm{I}=(-1)^{1 / 2}, k_{1}=i \pi, k_{2}=j \pi, i=1, \ldots, N-1, j=1, \ldots, M-1$. The amplification factor is written in the form $\rho=\mathrm{A}$. B and both $\mathrm{A}$ and $\mathrm{B}$ are expressions of the form numerator (U) divided by denominator (D), where $\mid$ Real $\mathrm{U} \mid \leq$ Real D and $\operatorname{Im} U=-\operatorname{Im}$ D. So, $|A| \leq 1$ and $|B| \leq 1$ and, therefore, the PRADI scheme is unconditionally stable.

\section{STABILITY OF THE GENERALIZED ADI SCHEME FOR THE VORTICITY EQUATION}

As shown by Dai, the main drawback of PRADI scheme is that the amplification factor $\mid \rho\left(\lambda_{x}, \lambda_{y},(N-\right.$ 1) $\pi,(N-1) \pi \mid \sim 1$ for large values of $\lambda_{x}$ and $\lambda_{y}$, implying that, for low Reynolds numbers, the solution obtained by PRADI scheme converges slowly to the solution of parabolic differential equation. Based on Dai's analysis, we can write the generalized ADI scheme for the vorticity equation as follows

$$
\begin{aligned}
& \left(1-\varepsilon_{x} \frac{\Delta t}{2 \operatorname{Re} h_{x}^{2}} \delta_{x}^{2}\right) \frac{\omega_{i j}^{n+1 / 2}-\omega_{i j}^{n}}{\Delta t / 2}=-\frac{u_{i j}^{n}}{2 h_{x}} \delta_{x} \omega_{i j}^{n+1 / 2} \\
& -\frac{v_{i j}^{n}}{2 h_{y}} \delta_{y} \omega_{i j}^{n}+\frac{1}{\operatorname{Re} h_{x}^{2}} \delta_{x}^{2} \omega_{i j}^{n+1 / 2}+\frac{1}{\operatorname{Re} h_{y}^{2}} \delta_{y}^{2} \omega_{i j}^{n} \\
& \left(1-\varepsilon_{y} \frac{\Delta t}{2 \operatorname{Re} h_{y}^{2}} \delta_{y}^{2}\right) \frac{\omega_{i j}^{n+1}-\omega_{i j}^{n+1 / 2}}{\Delta t / 2}=-\frac{u_{i j}^{n}}{2 h_{x}} \delta_{x} \omega_{i j}^{n+1 / 2}, \\
& -\frac{v_{i j}^{n}}{2 h_{y}} \delta_{y} \omega_{i j}^{n+1}+\frac{1}{\operatorname{Re} h_{x}^{2}} \delta_{x}^{2} \omega_{i j}^{n+1 / 2}+\frac{1}{\operatorname{Re} h_{y}^{2}} \delta_{y}^{2} \omega_{i j}^{n+1}
\end{aligned}
$$

where $\varepsilon_{x}$ and $\varepsilon_{y}$ are positive constants. As mentioned by Dai, when $\varepsilon_{x}=\varepsilon_{y}=0$, Eqs. (14a) and (14b) become the PRADI scheme. For the generalized scheme, the amplification factor is expressed by

$$
\begin{gathered}
\rho=\frac{1-\left(\lambda_{x} \beta_{x}-\lambda_{y} \varepsilon_{y} \beta_{y}\right)-\mathrm{I} \frac{\sigma_{x}}{2} \alpha_{x}}{1+\lambda_{x} \beta_{x}\left(1+\varepsilon_{x}\right)+\mathrm{I} \frac{\sigma_{x}}{2} \alpha_{x}} . \\
\frac{1-\left(\lambda_{y} \beta_{y}-\lambda_{x} \varepsilon_{x} \beta_{x}\right)-\mathrm{I} \frac{\sigma_{y}}{2} \alpha_{y}}{1+\lambda_{y} \beta_{y}\left(1+\varepsilon_{y}\right)+\mathrm{I} \frac{\sigma_{y}}{2} \alpha_{y}},
\end{gathered},
$$

where $\beta_{x}=1-\cos \left(k_{1} h_{x}\right), \quad \beta_{y}=1-\cos \left(k_{2} h_{y}\right), \quad \alpha_{x}=\sin \left(k_{l} h_{x}\right)$ and $\alpha_{y}=\sin \left(k_{2} h_{y}\right)$. This generalized scheme is unconditionally stable if $\lambda_{x} \beta_{x}-\lambda_{y} \varepsilon_{y} \beta_{y}>0$ and $\lambda_{y} \beta_{y}-\lambda_{x} \varepsilon_{x} \beta_{x}>0$. Since $\beta_{x}$ is either of $\mathrm{O}(1)$ or $\mathrm{O}\left(h_{x}^{2}\right)$, and $\beta_{y}$ is either of $\mathrm{O}(1)$ or $\mathrm{O}\left(h_{y}{ }^{2}\right)$, then $\varepsilon_{x}$ and $\varepsilon_{y}$ are $\mathrm{O}\left(h_{x}{ }^{2}\right)$ and $\mathrm{O}\left(h_{y}{ }^{2}\right)$, respectively. If $\lambda_{x}$ and $\lambda_{y}$ are very large so that $\lambda_{x} \beta_{x}$ and $\lambda_{y} \beta_{y}$ are significantly large, the modulus of the amplification factor tends to a value smaller than one. Therefore, this scheme is suited to simulate problems in which the Reynolds number is much smaller than one (creeping flows). The ADI method is conditionally stable at each 


\section{CIÊNCIA/SCIENCE}

time step (Sod, 1985). If one chooses $\lambda_{y} \beta_{y}-\lambda_{x} \varepsilon_{x} \beta_{x}>1$, then the first step (14a) may become unstable, and $\left\|\omega_{i j}^{n+1 / 2}\right\|_{2}$ may become large compared to $\left\|\omega_{i j}^{n}\right\|_{2}$ and $\left\|\omega_{i j}^{n+1}\right\|_{2}$. However, this is corrected when the second step (14b) is applied. So, for low Reynolds number problems, the time step should not have very large values.

Using the vorticity computed by Eqs. (11) or (14), the stream function (Eq. 2b) can be approximated by the PR-ADI scheme at the points $\left(x_{i}, y_{j}, t^{n+1 / 2}\right)$ and $\left(x_{i}\right.$, $y_{j}, t^{n+1}$ ) respectively as

$$
\begin{aligned}
& \frac{\psi_{i j}^{n+1 / 2}-\psi_{i j}^{n}}{\Delta t / 2}=\frac{\gamma}{h_{x}^{2}} \delta_{x}^{2} \psi_{i j}^{n+1 / 2}+\frac{\gamma}{h_{y}^{2}} \delta_{y}^{2} \psi_{i j}^{n}+\gamma \omega_{i j}^{n+1 / 2}, \\
& \frac{\psi_{i j}^{n+1}-\psi_{i j}^{n+1 / 2}}{\Delta t / 2}=\frac{\gamma}{h_{x}^{2}} \delta_{x}^{2} \psi_{i j}^{n+1 / 2}+\frac{\gamma}{h_{y}^{2}} \delta_{y}^{2} \psi_{i j}^{n+1}+\gamma \omega_{i j}^{n+1} .
\end{aligned}
$$

The components of the velocity vector in the interior of the rectangular region are computed by

$$
\begin{aligned}
& u_{i j}=\frac{\psi_{i j+1}-\psi_{i j-1}}{2 h_{y}}, \\
& v_{i j}=-\frac{\psi_{i+1 j}-\psi_{i-1 j}}{2 h_{x}} .
\end{aligned}
$$

\section{TIME-STEPPING ALGORITHM}

A time-stepping algorithm is developed through the use of Eqs (14), (16), and (17) for computing vorticity, stream-function and velocity vector.

\footnotetext{
Algorithm 1

1. Given Initial Conditions for $u^{0}, v^{0}, \omega^{0}$, and $\psi^{0}$

2. For $n=1,2, \ldots$ do:

3. $t^{n}=t^{n-1}+\Delta t$;

4. Compute $\omega^{n}$ using Eqs.(14) and $u^{n-1}, v^{n-1}$, and $\omega^{n-1}$

5. Compute $\psi^{n}$ using Eqs. (16) and $\omega^{n}$, and $\psi^{n-1}$

6. Compute $u^{n}$ and $v^{n}$ using Eqs. (17a) and (17b), respectively, and $\psi^{n}$

7. Update boundary conditions according described in section 2.

8. End of time iterations.
}

In the above algorithm, starting from initial conditions, the fields are computed independently of use of PR-ADI or generalized PR-ADI schemes. These are defined by the parameters $\varepsilon_{x}$ and $\varepsilon_{y}$.

\section{NUMERICAL RESULTS}

We compare the time-marching PR-ADI (Eq. 11) and the generalized PR-ADI (Eq. 14) schemes in the simulation of incompressible viscous flows. The NavierStokes equations are solved in a rectangular driven cavity using the stream function - vorticity approach. The vector velocity on the top is $\mathbf{V}=(u, v)=\left(4 x^{2}\left(1-x^{2}\right), 0\right)$ and the maximum of $u$ is $u_{\max }=1$ at $x=1 / 2^{1 / 2}$. This problem is solved using Eq. (2). The dependent variables $u, v, \omega$, and $\psi$ are determined by the time-marching Algorithm 1 .
H. A. Navarro and V. G. Ferreira A Numerical Scheme...

The time step is constant and the constant $\gamma=1$ is used. The space increments are computed as $h_{x}=x_{\max } / N$ and $h_{y}=y_{\max } / M$.

We consider a grid $N=20, M=20$. The time step is $\Delta t=10^{-4}$ and the cavity is square $(\mathrm{AR}=1)$. Table 1 shows the number of time steps for several Reynolds numbers. The number of time steps is reached when the time-independent boundary conditions forces a steady state, i. e., when the error for the vorticity $\left|\left(\omega_{i j}^{n+1}-\omega_{i j}^{n}\right) / \omega_{i j}^{n}\right|$ is smaller than a given tolerance TOL, at the grid points $(i, j), i=1, \ldots, N-1$ and $j=1, \ldots, M-1$. This table compares the two schemes (PR-ADI and generalized PR-ADI). When $\varepsilon_{x}=\varepsilon_{y}=0$, we observe that for very low Reynolds numbers $\left(\mathrm{Re} \leq 10^{-8}\right)$, the number of time steps increases. In the other case, for $\varepsilon_{x}=\varepsilon_{y}=0.0024$ smaller than $\mathrm{O}\left(h_{x}^{2}\right)$ and $\mathrm{O}\left(h_{y}{ }^{2}\right)$, it is noticeable that for Re $\leq 10^{-6}$ the number of time steps decreases. For Reynolds numbers greater than $10^{-3}$, the number of time steps computed by both schemes is the same. Nevertheless, for low Reynolds numbers, besides the number of time steps increasing when the PR-ADI scheme is used, we need to use a small tolerance for the vorticity $\left(\mathrm{TOL}=10^{-5}\right)$. Table 1 illustrates for $\mathrm{Re}=10^{-100}$ that the solution is reached with only 1,581 time steps when the generalized PR-ADI is used. The results obtained for the various small values of the Reynolds numbers $\left(\operatorname{Re} \leq 10^{-1}\right)$ are similar and show a convergence to $R e=0$. These small values of Reynolds were used only to simulate a Creeping Flow, where viscous effects predominate and inertia is negligible.

Table 1. Number of time steps for the schemes PR-ADI and generalized PR-ADI.

\begin{tabular}{cccc}
\hline Re & \multicolumn{3}{c}{ Number of time steps } \\
& \multicolumn{3}{c}{$\varepsilon_{x}=\varepsilon_{y}=0 / \varepsilon_{x}=\varepsilon_{y}=0.0024$} \\
& TOL $=10^{-3}$ & TOL $=10^{-4}$ & TOL $=10^{-5}$ \\
\hline 100 & $21,729 /$ & $67,813 /$ & $188,932 /$ \\
& 21,729 & 67,813 & 188,932 \\
10 & $7,359 /$ & $10,052 /$ & $13,840 /$ \\
& 7,359 & 10,052 & 13,840 \\
1 & $2,700 /$ & $2,772 /$ & $3,908 /$ \\
& 2,700 & 2,772 & 3,908 \\
$10^{-1}$ & $1,407 /$ & $1,770 /$ & $2,153 /$ \\
& 1,407 & 1,770 & 2,153 \\
$10^{-2}$ & $1,523 /$ & $1,932 /$ & $2,368 /$ \\
& 1,523 & 1,932 & 2,368 \\
$10^{-3}$ & $1,531 /$ & $1,943 /$ & $2,383 /$ \\
& 1,531 & 1,943 & 2,383 \\
$10^{-4}$ & $1,523 /$ & $1,928 /$ & $2,360 /$ \\
& 1,526 & 1,933 & 2,366 \\
$10^{-5}$ & $1,338 /$ & $2,336 /$ & $3,293 /$ \\
& 1,493 & 1,864 & 2,258 \\
$10^{-6}$ & $8,343 /$ & $13,900 /$ & $17,886 /$ \\
& 1,453 & 1,610 & 1,659 \\
$10^{-7}$ & $23,758 /$ & $82,986 /$ & $104,253 /$ \\
& 1,553 & 1,688 & 1,721 \\
$10^{-8}$ & $5,234 /$ & $229,571 /$ & $829,419 /$ \\
& 1,578 & 1,716 & 1,750 \\
$10^{-100}$ & $-/$ & $-/$ & $-/$ \\
& 1,581 & 1,719 & 1,754 \\
& & &
\end{tabular}




\section{CIÊNCIA/SCIENCE}

Figure 1 shows the stream function for $\mathrm{Re}=10^{-8}$. The stream function in Fig. 1(a) and (b) converge to different states from the ones reached in Fig. 1(c) and (d). Notice that Fig. 1(b) is slightly different near the top boundary. However, the number of time steps in Fig. 1(c) is much greater than in Fig. 1(d). Figure 1(d) presents the results obtained with the generalized PR-ADI showing that the solution is reached with a tolerance $\left(\mathrm{TOL}=10^{-3}\right)$ greater than that for the PR-ADI scheme $\left(\mathrm{TOL}=10^{-5}\right.$, Fig.1(c)). In Fig. 1(a) the tolerance was reached, but the solution does not present the same pattern because of a smaller number of time steps $(5,234-$ see Table 1$)$. This is a problem of the PR-ADI scheme for low Reynolds numbers. Using the same tolerance, the generalized PRADI scheme (Fig. 1(d)) efficiently captures the steadystate solution. It happens because the amplification factor tends to a value smaller than one for low Reynolds number, differently from the PR-ADI scheme (Fig. 1(a)) that tends to one.
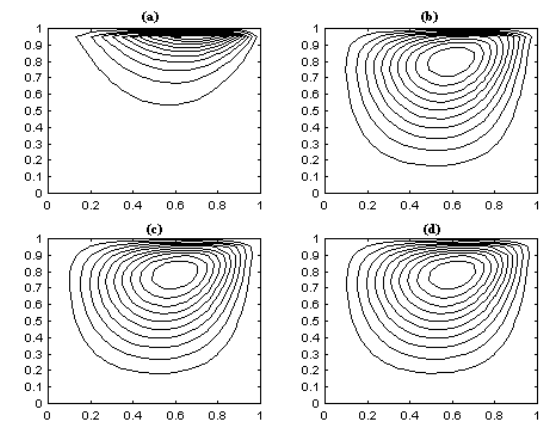

Fig. 1. Stream function contours for $\mathrm{Re}=10^{-8}, \Delta t=10^{-4}$ and $N=M=20$ : (a) $\mathrm{TOL}=10^{-3}, \varepsilon_{x}=\varepsilon_{y}=0$; (b) $\mathrm{TOL}=10^{-4}, \varepsilon_{x}=\varepsilon_{y}=0$; (c) $\mathrm{TOL}=10^{-5}, \varepsilon_{x}=\varepsilon_{y}=0$; (d) $\mathrm{TOL}=10^{-3}, \varepsilon_{x}=\varepsilon_{y}=0.0024$.

We now study what happens if the flow is in a rectangular cavity $(\mathrm{AR}=2)$. A grid $N=50, M=50$ is defined. In this case, the Reynolds number is $\mathrm{Re}=10^{-8}$, the time step is $\Delta t=10^{-5}$, and $\varepsilon_{x}=0.00039, \varepsilon_{y}=0.0015$. For a tolerance of $\mathrm{TOL}=10^{-3}$, the number of time steps is 19,017. Figure 2(a) illustrates that the stream function rotates in the clockwise direction in the superior part of the cavity. We can see the counterclockwise recirculation in the bottom of the cavity. Figure 2(b) shows the vorticity contours in the superior and inferior part of the rectangular cavity.
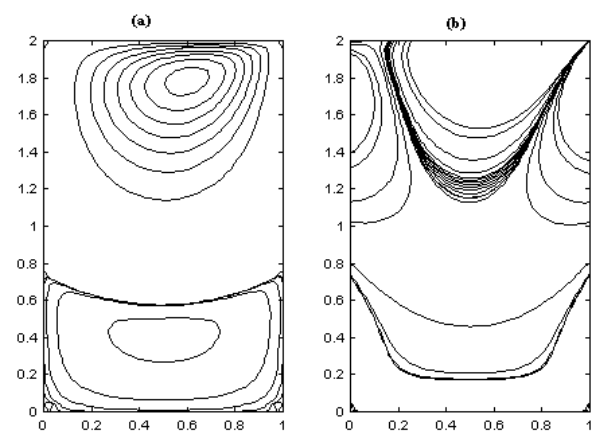

Fig. 2. Rectangular cavity $(\mathrm{AR}=2)$ : (a) stream function $\psi$, (b) vorticity $\omega$.
H. A. Navarro and V. G. Ferreira A Numerical Scheme... CONCLUSIONS

This paper has investigated the behavior of the generalized Peaceman-Rachford ADI scheme. This scheme is appropriate for low Reynolds number flow problems because of its rapid convergence characteristic. It has proved to be an efficient method for simulating fast transient phenomena and capturing steady-state solutions. We obtain good results for $\mathrm{Re}=10^{-8}$ and smaller values. As in each time step the ADI method is conditionally stable, choosing a very large $\Delta t$ is not recommended. We applied the generalized scheme in a cavity and the grid independence was confirmed. This method was also applied for a rectangular cavity and low Reynolds numbers. In this case, it was observed that a re-circulation in the inferior part of the cavity was obtained with few time steps. For high Reynolds number problems, both schemes (ADI and generalized) lead to the same results. 


\section{REFERENCES}

Burggraf, O.R., 1966, "Analytical and numerical studies of the structure of steady separated flows", J Fluid Mech, part 1; Vol.24, pp.113-151.

Dai, W., 1997, "A generalized Peaceman-Rachford ADI scheme for solving two-dimensional parabolic differential equations", J Sci Comput, Vol.12, No.4, pp.353-360.

Dean, J, Glowinski, R., 1993, "On some finite element methods for the numerical simulation of incompressible viscous flow. In: Incompressible Computational Fluid Dynamics", Cambridge, UK: University Press.

Ghia, U, Ghia, K.N., Shin, C.T., 1982, "High-Re solutions for incompressible flow using the NavierStokes equations and multigrid method", J Comput Phys, Vol.48, pp.387-411.

Glowinski, R., 1987, "Le $\theta$ scheme. In: Numerical Methods for the Navier-Stokes Equations", Bristeay M.O., Glowinski R., Perieux J. editors, Comput Phy Report, Vol.6, pp.73-187.

Hoffmann, K.A., Chiang, S.T., 1995, "Computational fluid dynamics for engineers". Engineering Education System, Vols 1 and 2.

Napolitano, M, Pascazio, G, Quartapelle, L., 1999, "A review of vorticity conditions in the numerical solution of the $\zeta-\psi$ equations", Computers \& Fluids, Vol.28, pp.139-185.

Pan, F., Acrivos, A., 1967, "Steady flows in rectangular cavities", J Fluid Mech, part 4; Vol.28, pp.643-655.

Peaceman, D., Rarchford, M., 1955, The numerical solution of parabolic and elliptic differential equations", J SIAM, Vol.3, pp.28-41.

Roache, P.J., 1972. "Computational fluid dynamics", Albuquerque: Hermosa.

Samarskii, A.A., Vabishchevich, P.N., 1994, "Regularized difference schemes", MMCE 1994, Vol.1, pp.44-50.

Sod, G.A., 1985, "Numerical methods in fluid dynamics: initial and initial boundary-value problems", Cambridge, UK: University Press.

Tannehill, J.C., Anderson, D.A., Pletcher, R.H.., 1997, "Computational fluid mechanics and heat transfer (Series in Computational and Physical Processes in Mechanics and Thermal Sciences)", Washington, DC: Taylor \& Francis.

Widllund, O.B., 1967, "On difference methods for parabolic equations and alternating direction implicit methods for elliptic equations", IBM J, pp.239-243. 